\title{
Hydrogel and Finger Caps: A Novel Dressing Method for Composite Grafts of Fingertips
}

\author{
Sam Yong Lee, Dong Gyu Lee, Jae Ha Hwang, Kwang Seog Kim \\ Department of Plastic and Reconstructive Surgery, Chonnam National University Medical School, Gwangju, Korea
}

\begin{abstract}
Background: Composite grafting is a useful reconstruction method for amputated fingertips when the size of the defect is small and vessels for anastomosis are not available. However, the survival rate of a composite graft is highly variable and difficult to anticipate. The most important factor for optimal wound healing is a moist environment, while wound desiccation is one of the main obstacles in graft survival. In this article, we introduce a new dressing method for preventing graft desiccation and enhancing the survival rate of grafts.

Methods: From February 2012 to February 2019, 18 patients with fingertip injuries distal to the distal interphalangeal joint with or without nail bed injury were enrolled. After the amputated part was reattached as a composite graft using 5-0 nylon, Duoderm Hydroactive Gel was applied on the graft, which was then covered with a "finger cap." Each finger cap was cut from a sterile latex glove. Including the degree of graft survival, any complications related to the graft were recorded.

Results: Not a single case of infection was observed. From the total of 18 composite grafts, eight took well without necrosis. In 10 cases, though partial necroses were observed they were of insignificant sizes, and all healed by secondary intention without other additional surgical procedures.

Conclusion: Treating fingertip defects with a composite graft and dressing method using hydrogel with a finger cap can prevent wound desiccation, enhance survival rates, and reduce patient distress.
\end{abstract}

Keywords: Tissue transplantation; Finger; Wound healing

\section{Introduction}

The fingertips are the human body area most frequently injured [1]. When reconstructing amputated fingertips, replantation by microvascular anastomosis is the treatment of choice. In situations where replantation cannot be performed, several other options are available, including stump revision, skin or composite grafting, and local and free flaps. If no suitable vessels for anastomosis are available, composite grafting could be a good option for restoring aesthetic appearance, sensation, and function of the fingertip without multiple time-consuming procedures. However, the success rates of composite grafting are highly variable and often unpredictable [2-4].

A moist environment is well known to be an important factor in wound healing [5]. Moist treatment of wounds enhances wound healing, boosts re-epithelialization, lowers rate of infection, promotes autolytic debridement and reduces scarring. On top of all these benefits, moisture prevents wound desiccation. In addition, cytokine and growth factors trigger beneficial effects and promote angiogenesis and revascularization [6-12].

A wet environment around a wound prevents desiccation, and thereby prevents necrotic tissue from developing on the surface of the wound. We therefore assumed that a dry environment would promote graft desiccation and increase the incidence of

\section{Original Article}

Received: August 9, 2019

Revised: September 11, 2019

Accepted: September 11, 2019

\section{Corresponding author: \\ Jae Ha Hwang, M.D., Ph.D.}

Department of Plastic and Reconstructive Surgery, Chonnam National University Medical School, 42 Jebong-ro, Dong-gu, Gwangju 61469, Korea

Tel: +82-62-220-6363

Fax: +82-62-220-6357

E-mail: psjhhwang@daum.net

This is an Open Access article distributed under the terms of the Creative Commons Attribution Non-Commercial License (http://creativecommons.org/licenses/by-nc/4.0/) which permits unrestricted non-commercial use, distribution, and reproduction in any medium, provided the original work is properly cited.

(c) 2019 Korean Wound Management Society 
graft necrosis. Before revascularization, the graft is nourished by plasma diffused from the wound bed [13]. The longer the graft is protected from desiccation, the further will the host vessels from the wound bed grow into the graft, increasing the likelihood of survival for the graft. In the authors' experience, conventional compression dressing with petroleum gauze does not guarantee adequate hydration, often resulting in desiccation of grafts and then graft necrosis. In this article, we introduce a new dressing method to maintain moisture, prevent desiccation of grafts and ultimately increase their chances of survival.

\section{Methods}

We conducted a retrospective chart review of patients who underwent composite graft of the fingertip between February 2012 and February 2019. The study was performed in accordance with the principles of the Declaration of Helsinki. As a retrospective review of medical records, the study itself did not require informed consent; written informed consent was obtained from the patients for the use and publication of their images. Patients who were injured proximal to the distal interphalangeal joint, those with bone exposure, contaminated wounds, autoimmune disease and those receiving dialysis were excluded. The following items were investigated: age, sex, site of injury, size of defect, mechanism of amputation, any documented complications, and the degree of graft survival.

Mechanisms of amputation were recorded as laceration, crushing or avulsion. Lacerations were defined as injuries from sharp objects, without tissue loss. Crushing was from blunt objects with some loss and crushing of tissues. Avulsion injuries were those with severe crushing or avulsion of tissues [14]. Graft survival was also grouped into three categories: complete, incomplete, and failed. Incomplete graft survival was defined as any graft on which there were patches of necrotic tissue interspersed with viable tissues. Failed graft was defined as total tissue necrosis at the tip of the finger [15].

For all the patients, fingertip composite grafting was performed under digital block of the affected digit using $2 \%$ lidocaine. After applying a finger tourniquet, the wound and graft were irrigated thoroughly. Foreign bodies and devitalized tissues were debrided. The size of the defect was measured intraoperatively in length and width. After electrocauterizing to control bleeding, the amputated part was reattached as a composite graft using 5-0 nylon (Fig. 1). Duoderm Hydroactive Gel (Convatec, E.R. Squibb \& Sons Inc., Princeton, NJ, USA) was then applied on the graft and covered with a "finger cap," cut from a sterile latex glove (Fig. 2). After the composite graft was covered with the finger cap, the cap was held in place at the proximal end with gauze and elastic bandages to keep the gel from oozing out. The dressing, including the finger cap and hydrogel, was changed daily for the first 3 days after surgery.
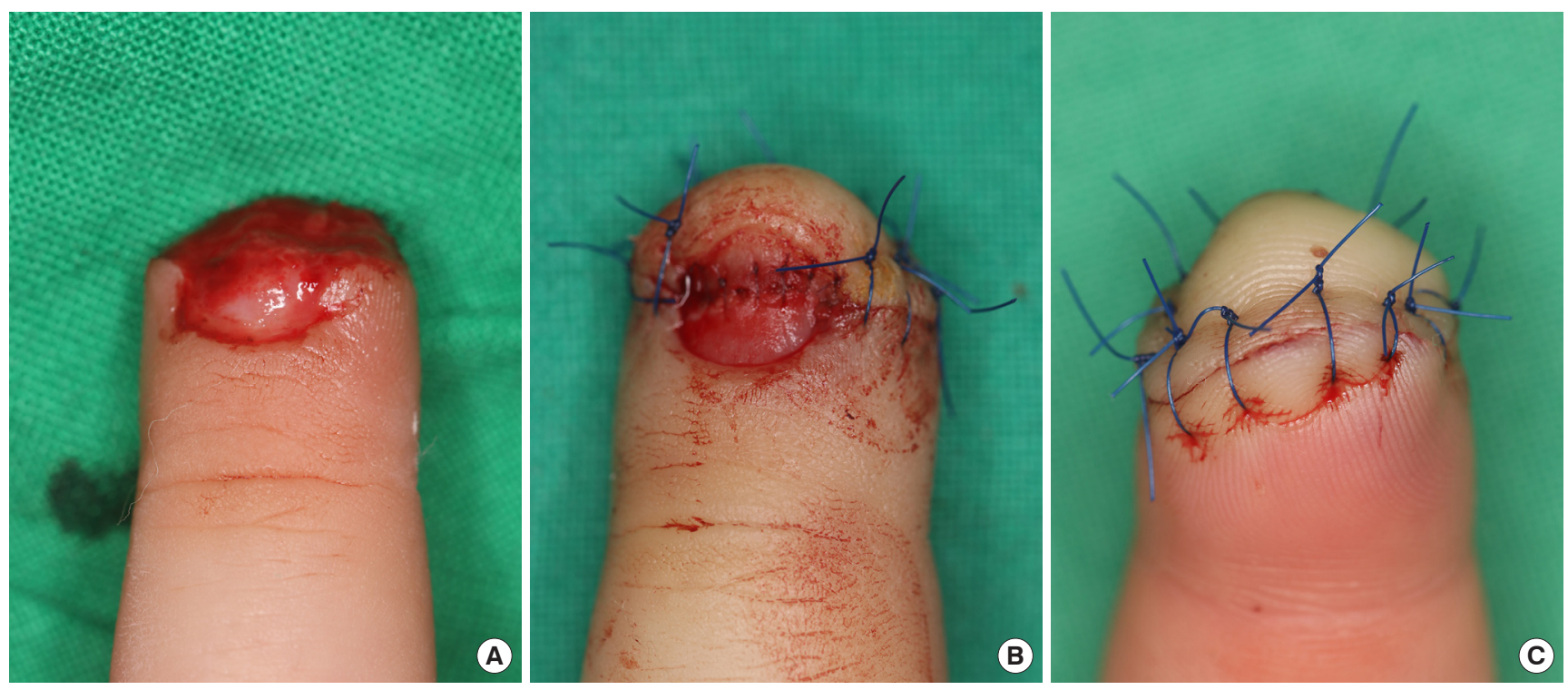

Fig. 1. Photographs of patient no. 5. (A) A 2-year-old male patient was presented to the emergency department with a fingertip injury that required amputation of his left middle finger. His finger was injured after being trapped and crushed in a door. After debridement and irrigation, a composite graft of the fingertip was sutured with 5-0 nylon. Dorsal (B) and volar (C) views. 
Afterwards, the frequency of dressing changes was adjusted depending on how well the graft was hydrated. The survival rates of the grafts were determined on the basis of the clinical photographs taken 4 weeks after the procedure (Figs. 3-5).

\section{Results}

Eighteen patients with 18 fingertips in total satisfied the inclusion criteria (Table 1). The patients' mean age was $29 \pm 19$ years (range, 2-58 years). Ten were male (56\%) and eight (44\%) were female. The most common mechanism of amputation was by crushing, observed in nine patients and the others were laceration in five patients and avulsion in four patients. The size of the fingertip defect ranged from $3 \times 3$ to $0.5 \times 0.2 \mathrm{~cm}^{2}$, and the mean area of the defect was $2.18 \pm 1.99 \mathrm{~cm}^{2}$.

None of the cases showed any signs of inflammation or infection. In eight patients, full graft survival was observed. In all of the other 10 patients, the grafts survived incompletely, but
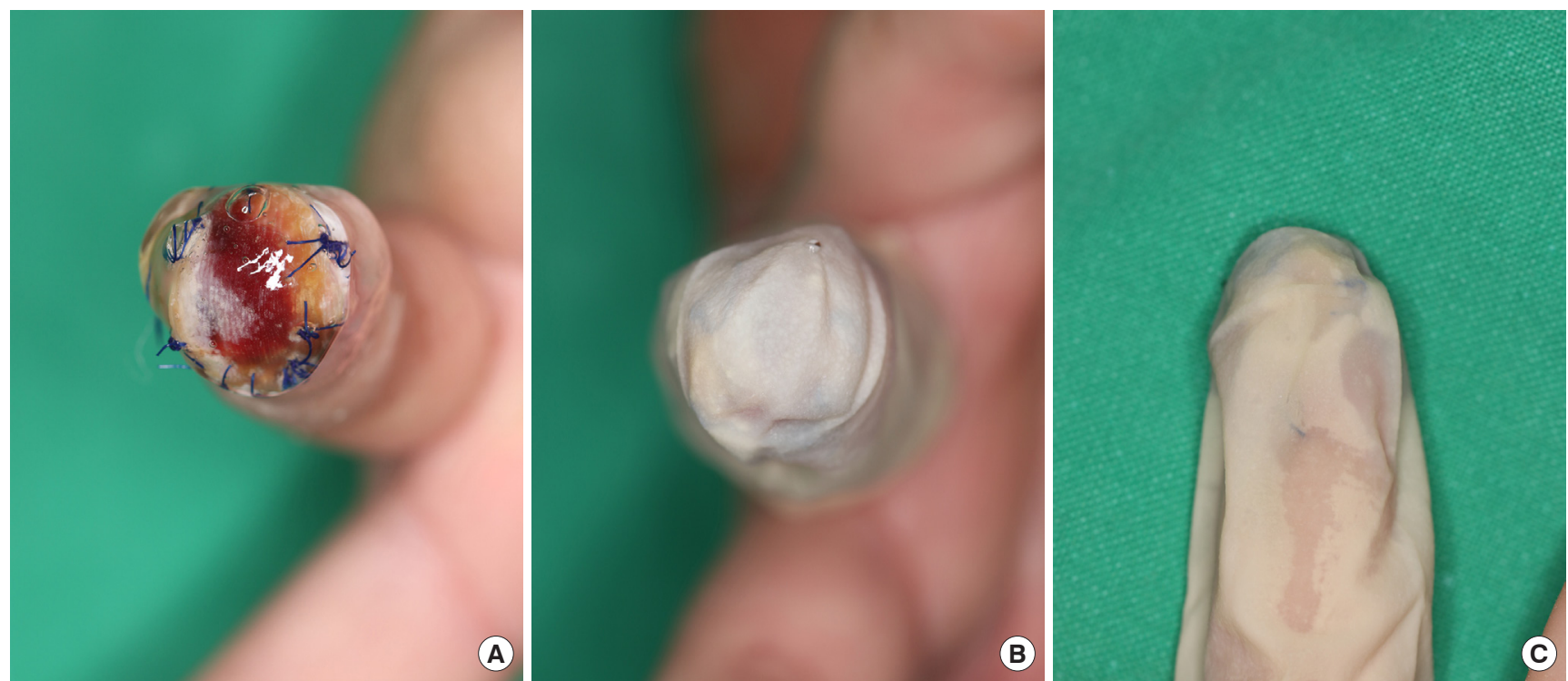

Fig. 2. Dressing method of patient no. 5. Amorphous hydrogel was applied on the graft (A), and the graft was covered with a finger cap tailored from a sterile latex glove (B, C).
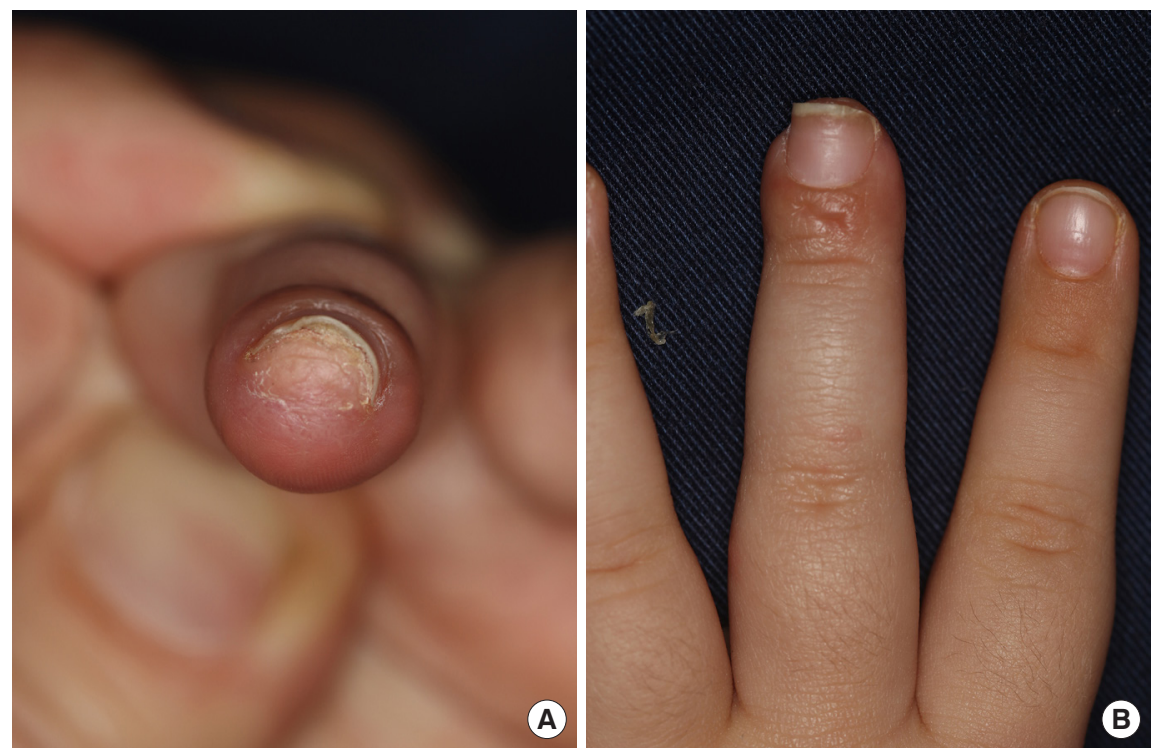

Fig. 3. Follow-up photographs of patient no. 5. (A, B) Clinical photographs 4 weeks after grafting. 

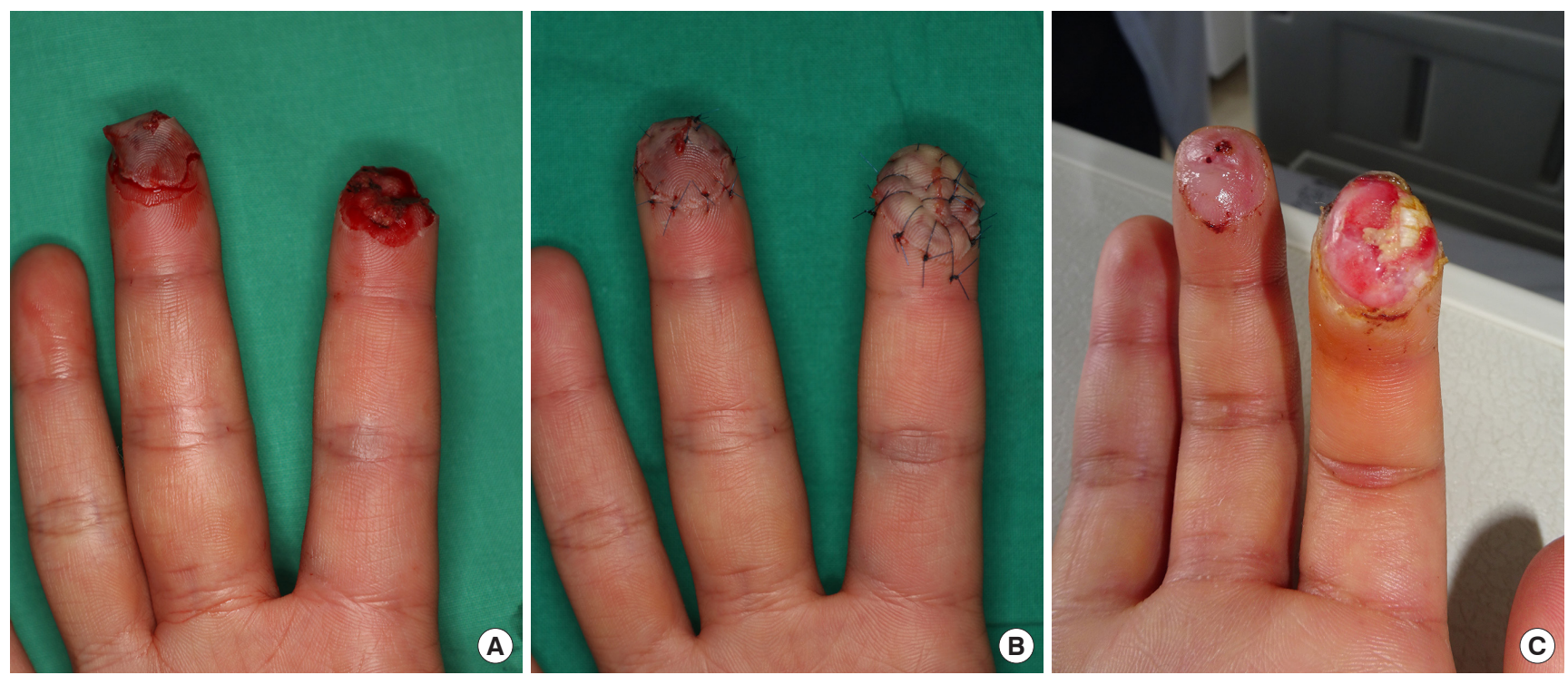

Fig. 4. Photographs of patient no. 6. (A) A 24-year-old female patient had her right index and middle finger crushed under a heavy object. (B) The avulsed part was reattached in a composite graft in the right index fingertip. (C) Clinical photograph 4 weeks after grafting. Slough is observed but gel dressing was sufficient for wound healing by secondary intention.
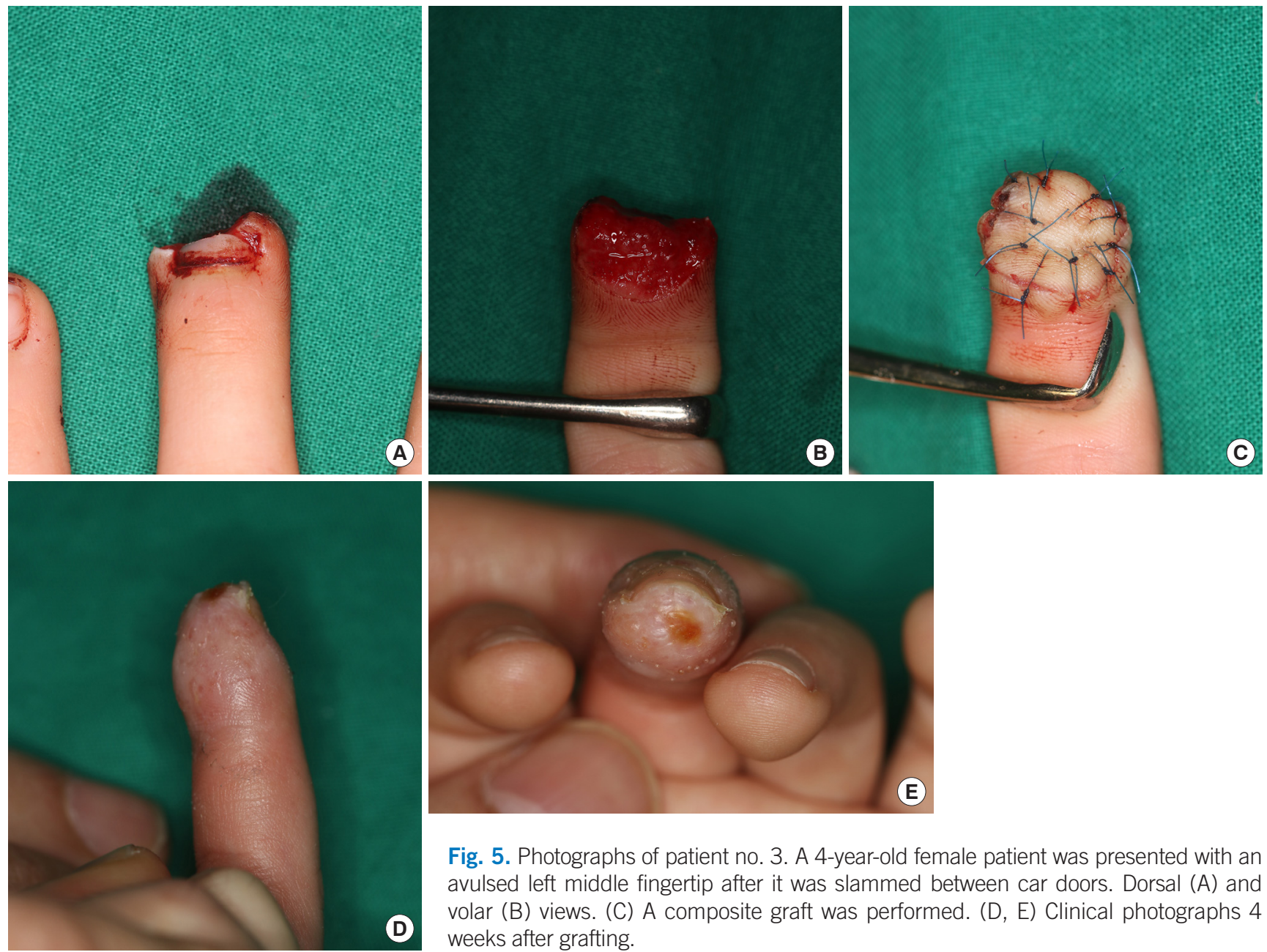

Fig. 5. Photographs of patient no. 3. A 4-year-old female patient was presented with an avulsed left middle fingertip after it was slammed between car doors. Dorsal $(A)$ and volar (B) views. (C) A composite graft was performed. (D, E) Clinical photographs 4 weeks after grafting. 
Novel dressing for fingertip composite grafts

Table 1. Patient demographics

\begin{tabular}{|c|c|c|c|c|c|c|c|}
\hline Patient no. & Age (yr) & Sex & Fingertip injured & Size of defect $\left(\mathrm{cm}^{2}\right)$ & Mechanism of amputation & Graft survival & Nailbed injury \\
\hline 1 & 2 & $\mathrm{~F}$ & Left little finger & $1 \times 1$ & Crush & Complete & Accompanied \\
\hline 2 & 35 & M & Left index finger & $1 \times 0.5$ & Laceration & Complete & Accompanied \\
\hline 3 & 4 & $\mathrm{~F}$ & Left middle finger & $1.5 \times 1.5$ & Crush & Incomplete & Accompanied \\
\hline 4 & 34 & $\mathrm{~F}$ & Left thumb & $0.5 \times 0.2$ & Laceration & Incomplete & Accompanied \\
\hline 5 & 2 & M & Left middle finger & $1 \times 1$ & Crush & Complete & Accompanied \\
\hline 6 & 24 & $\mathrm{~F}$ & Right index finger & $2 \times 2$ & Avulsion & Incomplete & Accompanied \\
\hline 7 & 58 & $\mathrm{~F}$ & Left thumb & $2 \times 1$ & Laceration & Incomplete & Accompanied \\
\hline 8 & 44 & M & Right thumb & $1 \times 1$ & Laceration & Incomplete & None \\
\hline 9 & 34 & M & Right middle finger & $2 \times 2$ & Crush & Incomplete & None \\
\hline 10 & 32 & M & Right thumb & $3 \times 3$ & Avulsion & Incomplete & None \\
\hline 11 & 46 & M & Right middle finger & $2 \times 1.5$ & Crush & Incomplete & None \\
\hline 12 & 42 & $\mathrm{~F}$ & Right ring finger & $1 \times 1$ & Crush & Complete & Accompanied \\
\hline 13 & 50 & M & Right index finger & $2 \times 1$ & Avulsion & Incomplete & Accompanied \\
\hline 14 & 41 & $\mathrm{~F}$ & Left index finger & $3 \times 1$ & Laceration & Complete & Accompanied \\
\hline 15 & 22 & M & Right index finger & $2 \times 1$ & Avulsion & Incomplete & None \\
\hline 16 & 53 & $\mathrm{~F}$ & Left little finger & $2 \times 1$ & Crush & Complete & Accompanied \\
\hline 17 & 3 & M & Left ring finger & $0.7 \times 0.7$ & Crush & Complete & None \\
\hline 18 & 3 & M & Right middle finger & $1 \times 1$ & Crush & Complete & Accompanied \\
\hline
\end{tabular}

F, female; M, male.

the portion of the necrosis was insignificant (i.e., all necroses amounted to less than $50 \%$ of the size of the total defect). Healing by secondary intention was sufficient for all of them and no subsequent surgical procedures were necessary.

In 12 patients (67\%), the defect was accompanied by a nail bed injury. None of them manifested complications or deformities related to the nail bed, including non-adherence, nail plate deformity, or hooked nail.

\section{Discussion}

In the 1960s, Winter first demonstrated the importance of a moist environment in wound healing [5]. Since then, the numerous benefits of wound hydration and moist wound healing have been proved and accepted as a basic principle in modern wound care. Moist wound treatment has various advantages: it boosts wound healing, enhances re-epithelialization, lowers rate of infection, promotes autolytic debridement, delivers good cosmesis by reducing scars, and allows prolonged presence of growth factors and cytokines which lead to favorable outcomes through wound contraction and angiogenesis promotion [5-12].

Revascularization is critical for graft survival. Before revas- cularization, the graft attains oxygen and nutrients through diffusion from plasma between the graft and the wound bed [16]. Revascularization starts as early as 2 days post-grafting and vessel growth occurs at about $5 \mu \mathrm{m} / \mathrm{hr}[13,17]$. The thicker the grafts are, the harder it is for them to survive. Before angiogenesis is complete, graft survival purely depends on exudates from the wound bed. During this period, any desiccation will exacerbate graft necrosis and contribute to graft failure. Keeping the wound environment moist prevents a graft from desiccating and delays graft necrosis until the blood vessels are reconnected. The authors therefore hypothesized that a moist environment would eventually increase the graft survival rate. This had been demonstrated in the case of an upside-down split-thickness skin graft in a wet environment [18]. Even though the split-thickness skin graft was transplanted inverted (with the stratum corneum facing the wound bed), it could successfully survive if a wet wound environment was provided. In a dry wound environment, on the other hand, inverted grafts did not survive and showed significantly lower re-epithelialization. Zuhaili et al. [18] described that the successful outcome of the research owed to wound hydration and emphasized the importance of the wound environment in regeneration. 
In our experience, when performing conventional dressings using topical antibacterial ointment and petroleum gauze, graft desiccation starts 5 to 10 days post-grafting. However, no graft desiccation was observed at 14 days after the procedures in all of the 18 cases. On the contrary, the grafts were hyperhydrated and became white and wrinkly. At first glance, hyper-hydration of the skin looks similar to moisture-associated skin damage (MASD). MASD is mainly due to chronic wound exudates containing protein-degrading enzymes, elevated proinflammatory cytokines and increased neutrophils $[19,20]$. Combined with proteases produced from bacteria, these exudates have corrosive effects on peri-wound skin [21]. The soft tissue defects observed in this study were grafted with the amputated parts in the acute stage and thus were different from the chronic wound environments characterized by prolonged inflammation which MASD is generally associated with. Besides, unlike the corrosive effect of MASD, hyper-hydration is limited to the stratum corneum. It is reversible and the skin itself is not excessively damaged [22]. In the authors' experience, hyper-hydration due to continued exposure to the hydroactive gel over 2 weeks was harmless to the adjacent skin as well as the composite graft itself.

Although the authors are convinced of the effects of a moist environment in composite grafting, this study has some limitations. The absence of a control group made statistical comparisons impossible. In a single-center-based prospective study that included composite grafts in 57 digital tip amputations, $52.6 \%(\mathrm{n}=30)$ of all the composite grafts survived completely. Partial survival and graft failure was $31.6 \%(\mathrm{n}=18)$ and $15.8 \%(n=9)$ respectively. In that study, the authors concluded that smoking was the only significant factor that had a strong association with graft failure [23]. In a separate retrospective review of 100 patients under 16 years of age, $13 \%(n=13)$ survived completely, $46 \%(n=46)$ partially and $41 \%(n=41)$ failed [2]. Though a simple comparison of the present study and the former two studies would be far from significant, the fact that none $(0 \%)$ of the grafts in the current study failed while $13 \%$ and $41 \%$ had failed respectively in the two previous studies might underscore the effectiveness of moist wound treatment on fingertip composite grafts, or it could be attributed to selection bias. In general, because it is difficult for thicker and wider composite grafts to survive, local, distant or free flaps are preferred over composite grafts for larger defects. This results in a tendency to perform composite grafts only on smaller defects, which could have affected the outcome of the study. The authors plan to include a more controlled statistical analysis of the effects of a moist environment on composite grafts in a future study.

In a wet or moist wound environment under occlusive dressings, evaporation, loss of energy and desiccation are reduced [11]. Presence of growth factors and proteinases in fluid exudates is maintained, also contributing to the healing [24,25]. In cases of split-thickness skin grafts, exudates containing these nutrients and growth factors are sufficient to nourish the graft during serum imbibition. On the other hand, when it comes to composite grafts, the tissue is thick and it is difficult for exudates to reach the most superficial area of the graft. If the wound environment is wet, occluded chamber fluid reaches equilibrium with the wound exudates and contains concentrations of electrolytes, glucose and protein very similar to those in serum [25]. In the same way, if a moist occlusive environment is provided for the composite grafts, evaporation and energy loss are reduced, growth factors and nutrients are preserved and contribute to graft survival.

Compared to other parts of the body, the fingertip is a much more difficult area to dress because of its frequent movements in daily life and its own curvature. Using a finger cap tailored from sterile latex gloves which are readily available makes dressings much easier. Moreover, because the latex is waterproof, dressing once a day or every 2 or 3 days is enough to maintain adequate hydration of the graft. Such a dressing constitutes the "ideal," according to the British National Health System, in that it is impermeable to water and bacteria, minimizes the frequency of dressing changes, can absorb and contain exudate, provides thermal insulation, does not leave particulate contaminants in the wound, does not traumatize the wound bed when removed, and provides pain relief and comfort when used. By virtue of the amorphous hydrogel, this dressing does not come into contact with the wound bed and thus leaves nothing in the wound and does not traumatize the wound or cause discomfort. Although the authors had not specifically surveyed subjective or objective data on patient discomfort, very few patients complained about pain.

Various autologous and recombinant growth factors have been introduced as enhancing the wound healing process, including vascular endothelial growth factor, transforming growth factor, platelet-derived growth factor, polydeoxyribonucleotide, and platelet-rich plasma. With these growth factors, angiogenesis and damage from ischemia-reperfusion injuries of the grafts are improved and the graft survival rate could increase. These recombinant or autologous growth factors used in conjunction with wound hydration could pro- 
mote graft survival even further.

In wound healing, wound hydration is the single most important external factor; therefore hydrating composite grafts could prevent necrosis from graft desiccation and increase the rates of survival for grafts. In treating fingertip defects using composite grafts, dressing with hydrogel and a finger cap is useful, not only keeping grafts hydrated but also reducing the frequency of dressings, lessening patient discomfort, and preventing infections. We recommend this dressing technique for successful fingertip composite grafting.

\section{Conflict of interest}

No potential conflicts of interest relevant to this article are reported.

\section{Acknowledgments}

Sam Yong Lee https://orcid.org/0000-0002-3185-2519

Dong Gyu Lee https://orcid.org/0000-0003-3701-0395

Jae Ha Hwang https://orcid.org/0000-0001-6992-8067

Kwang Seog Kim https://orcid.org/0000-0002-6766-4640

\section{References}

1. Chen CT, Wei FC, Chen HC, et al. Distal phalanx replantation. Microsurgery 1994;15:77-82.

2. Borrelli MR, Dupre S, Mediratta S, et al. Composite grafts for pediatric fingertip amputations: a retrospective case series of 100 patients. Plast Reconstr Surg Glob Open 2018;6:e1843.

3. Huang HF, Yeong EK. Surgical treatment of distal digit amputation: success in distal digit replantation is not dependent on venous anastomosis. Plast Reconstr Surg 2015;135:174-8.

4. Chen SY, Wang CH, Fu JP, et al. Composite grafting for traumatic fingertip amputation in adults: technique reinforcement and experience in 31 digits. J Trauma 2011;70:148-53.

5. Winter GD. Formation of the scab and the rate of epithelization of superficial wounds in the skin of the young domestic pig. Nature 1962;193:293-4.

6. Eaglstein WH. Moist wound healing with occlusive dressings: a clinical focus. Dermatol Surg 2001;27:175-81.

7. Atiyeh BS, El-Musa KA, Dham R. Scar quality and physiologic barrier function restoration after moist and moist-exposed dressings of partial-thickness wounds. Dermatol Surg 2003; 29:14-20.

8. Erfurt-Berge C, Renner R. Recent developments in topical wound therapy: impact of antimicrobiological changes and rebalancing the wound milieu. Biomed Res Int 2014;2014:819525.

9. Atiyeh BS, Hayek SN. Interet d'un Onguent Chinois (MEBO) dans le Maintient Local de l'Humidite. J Plaies Cicatrisation 2005;9:7-11.

10. Mosti G. Wound care in venous ulcers. Phlebology 2013;28 Suppl 1:79-85.

11. Svensjo T, Pomahac B, Yao F, et al. Accelerated healing of fullthickness skin wounds in a wet environment. Plast Reconstr Surg 2000;106:602-12.

12. Hackl F, Kiwanuka E, Philip J, et al. Moist dressing coverage supports proliferation and migration of transplanted skin micrografts in full-thickness porcine wounds. Burns 2014;40: 274-80.

13. Clemmesen T, Ronhovde DA. Restoration of the blood-supply to human skin autografts. Scand J Plast Reconstr Surg 1968; 2:44-6.

14. Biemer E. Definitions and classifications in replantation surgery. Br J Plast Surg 1980;33:164-8.

15. Butler DP, Murugesan L, Ruston J, et al. The outcomes of digital tip amputation replacement as a composite graft in a paediatric population. J Hand Surg Eur Vol 2016;41:164-70.

16. Converse JM, Uhlschmid GK, Ballantyne DL Jr. "Plasmatic circulation" in skin grafts: the phase of serum imbibition. Plast Reconstr Surg 1969;43:495-9.

17. Zarem HA. The microcirculatory events within full-thickness skin allografts (homografts) in mice. Surgery 1969;66:392-7.

18. Zuhaili B, Aflaki P, Koyama T, et al. Meshed skin grafts placed upside down can take if desiccation is prevented. Plast Reconstr Surg 2010;125:855-65.

19. Moore K. Cell biology of normal and impaired healing. In: Percival S, Cutting K, editors. Microbiology of wounds. Boca Raton: CRC press; 2010. p. 151.

20. Yager DR, Chen SM, Ward SI, et al. Ability of chronic wound fluids to degrade peptide growth factors is associated with increased levels of elastase activity and diminished levels of proteinase inhibitors. Wound Repair Regen 1997;5:23-32.

21. Wildeboer D, Hill KE, Jeganathan F, et al. Specific protease activity indicates the degree of Pseudomonas aeruginosa infection in chronic infected wounds. Eur J Clin Microbiol Infect Dis 2012;31:2183-9.

22. Rippon MG, Ousey K, Cutting KF. Wound healing and hyperhydration: a counterintuitive model. J Wound Care 2016;25:6875.

23. Heistein JB, Cook PA. Factors affecting composite graft survival in digital tip amputations. Ann Plast Surg 2003;50:299- 
Lee SY et al.

Novel dressing for fingertip composite grafts

303.

24. Breuing K, Andree C, Helo G, et al. Growth factors in the repair of partial thickness porcine skin wounds. Plast Reconstr Surg 1997;100:657-64.
25. Vogt PM, Lehnhardt M, Wagner D, et al. Determination of endogenous growth factors in human wound fluid: temporal presence and profiles of secretion. Plast Reconstr Surg 1998; 102117-23. 